\title{
Reduction in healthcare utilization and costs following residential integrated treatment for co-occurring substance use and mental health disorders
}

\author{
Siobhan Morse ${ }^{1}$, Brian E. Bride*2 \\ ${ }^{1}$ Addiction Services Division/Universal Health Services, Foundations Recovery Network, United States \\ ${ }^{2}$ Georgia State University, United States
}

Received: August 12, 2016

DOI: $10.5430 /$ jha.v5n6p53
Accepted: September 7, 2016

Online Published: September 23, 2016

URL: http://dx.doi.org/10.5430/jha.v5n6p53

\begin{abstract}
Objective: The purpose of this study was to examine the impact of residential, integrated treatment for co-occurring substance use and mental health disorders on healthcare utilization and costs. More specifically, we compared pre- and post-treatment utilization of emergency room (ER) services and hospital stays for medical, mental health, and substance use problems.

Methods: Data from 1,394 patients who attended private, residential treatment for co-occurring disorders and participated in follow-up data collection at 6 months were analyzed using chi-square and $t$-tests to evaluate what changes, if any, occurred preand post-treatment in regard to ER and hospital overnight utilization.

Results: Significant reductions in the number ER visits and overnight stays were realized across the three services domains examined: medical, mental health and substance use.

Conclusions: Private, residential treatment for substance use and mental health disorder can support an overall reduction in the utilization and costs of acute care services such as ER visits and hospital overnight stays.
\end{abstract}

Key Words: Residential treatment, Co-occurring disorders, Integrated treatment, Healthcare utilization, Healthcare costs

\section{INTRODUCTION}

Over the past twenty years, several studies have estimated the economic burden that alcohol and drug abuse have placed on society. The National Institute on Drug Abuse reports $\$ 36$ billion in health care costs for individuals abusing alcohol and/or illicit drugs. ${ }^{[1]}$ These estimates include costs associated with crime, law enforcement and the judicial system, health care expenditures, and loss estimates such as lost productivity and the social costs of premature death. ${ }^{[2]}$ Health care costs include: (1) those that are associated with the diagnosis and treatment of alcohol/drug abuse; (2) costs related to associated disorders or medical issues, such as liver disease in an alcoholic or Hepatitis infection in intravenous drug users; as well as (3) additional health and medical care costs not directly associated with the alcohol or drug use but resulting from the unhealthy lifestyle. This latter category is rarely reported because of the difficulty in quantifying such a number.

A significant body of literature suggests that the health care costs of individuals who abuse drugs and alcohol are higher than those for the general populations. Individuals who abuse drugs and alcohol have higher rates of physical illnesses many of which are consequences of excessive alcohol or drug use, including infectious diseases, cardiovascular

*Correspondence: Brian E. Bride, Prof.; Email: bbride@ gsu.edu; Address: Georgia State University, United States. 
effects, respiratory effects, gastrointestinal effects, musculoskeletal effects, kidney damage, liver damage, neurological effects, mental health effects, hormonal effects, cancer, prenatal effects, other health effects, and mortality. ${ }^{[2,3]}$ It is estimated that of the $\$ 216$ billion dollars paid by federal and state government for health care costs for persons abusing alcohol, drugs and tobacco $98 \%$ represents medical consequences of substance abuse and only $2 \%$ represents direct spending for substance abuse treatment. ${ }^{[4]}$

Individuals with drug or alcohol use disorders use more expensive forms of acute care. ${ }^{[3]}$ Cederbaum and colleagues note an increased use of emergency room (ER) services by individuals suffering from mental health and substance use disorders. ${ }^{[5]}$ Chronic drug users utilize $30 \%$ more ER services than the general population. ${ }^{[6]}$ The Drug Abuse Warning Network (DAWN) reports nearly 4.6 million drug related ER visits in 2009, representing an $81 \%$ increase since 2004. ${ }^{[7]}$ Opiate users often receive care in ERs, one of the most expensive points of entry to the healthcare system. ${ }^{[8]}$ Oxycodone-based medications were the pain relievers most commonly involved in ER visits (56.2 visits per 100,000). ${ }^{[9]}$

Prescription drug users make up 55\% of the estimated 8.9 million drug users in the United States and hospitalizations for opiates are highest for prescription drugs. From 1993 through 2012, the rate of adult hospital stays due to opiate overuse increased by $5 \%$ annually. ${ }^{[10]}$ The hospitalizations included stays for overuse of opiates, including dependence, abuse, poisoning and adverse effects. ${ }^{[11]}$ The healthcare related costs of excessive alcohol consumption in 2006 was nearly $\$ 25$ billion. ${ }^{[12]}$

Individuals who abuse drugs and alcohol have higher rates of mental illnesses. ${ }^{[3]}$ According to the Substance Abuse and Mental Health Services Administration (SAMHSA), people with mental health disorders are more likely than people without mental health disorders to experience an alcohol or substance use disorder. ${ }^{[13]}$ There is a higher risk of hospitalization as well as ER visits in bipolar patients among those who also have substance abuse issues. ${ }^{[14]}$

The purpose of this study was to examine the impact of residential, integrated treatment for co-occurring substance use and mental health disorders on healthcare utilization and costs. Integrated treatment in this context refers to the practice of treating both substance use and mental health disorders simultaneously by the same clinicians, rather than in a sequential model in separate systems. More specifically, we compared pre- and post-treatment utilization of ER services and hospital stays for medical, mental health, and substance use problems.

\section{METHOD}

\subsection{Sample}

Data used for this analysis were collected from individuals who entered treatment in 2014 and 2015 at four residential facilities operated by Foundations Recovery Network, the Addiction Services Division of Universal Health Services. These treatment facilities provide integrated substance abuse and mental health treatment services to patients drawn from across the United States and Canada. Admission criteria are based on medical necessity determined by insurance or other third party payers and are inclusive of substance use history and use patterns, psychiatric symptoms, previous treatment experience, and take into account medical conditions, and the social and legal consequences of the patient's substance use. Of 2,418 individuals entering treatment between January 1, 2014 and February 10, 2015, 2,167 consented to participate in this research, representing a $90 \%$ enrollment rate. Of those who completed data at intake, 1,424 (66\%) completed the one-month follow-up, and 1,394 (64\%) completed the sixmonth follow-up. Study participants were primarily White (92\%), non-Hispanic (93\%), and male (57\%). Participant ages ranged from 18 to 73 , with a mean of $34.6(S D=13.60)$.

\subsection{Data collection}

Data was collected by trained interviewers in face-to-face meetings at intake, and by telephone interview at 30-days and 6-months post-discharge. Items concerning healthcare utilization were taken from the Addiction Severity Index ${ }^{[15]}$ and the Treatment Services Review. ${ }^{[16]}$ More specifically, at intake patients separately reported the number of ER admissions in the previous six months for medical, mental health, and substance abuse problems. In addition, patients reported the number of nights they stayed in the hospital for medical, mental health, and substance abuse problems. At the 30-day follow-up interview, patients reported ER visits and hospital stays since leaving residential treatment. At the sixmonth follow-up, patients reported ER visits and hospital stays since their last interview with us (i.e., 30-day followup). Because the U.S. lacks a comprehensive and integrated electronic health record system, it was necessary to utilize self-report data. Data from the 30-day and six-month interviews were summed within each category to obtain an estimate of ER visits and hospital stays in the six months following treatment, thus allowing a valid comparison window for the six-month pre-treatment estimates. Utilization costs for ER visits were calculated based upon an average cost of $\$ 1,579$ per ER admission as estimated by the Agency for Healthcare Research and Quality. ${ }^{[17]}$ Costs for hospital stays were calculated based on an average cost of $\$ 2,212$ per night as estimated by the Kaiser Family Foundation. ${ }^{[18]}$ 


\section{Results}

\subsection{Healthcare utilization}

Columns 2 and 5 of Table 1 display the number and percentage of individuals who reported ER visits and/or inpatient stays for medical, mental health, and substance use problems for the six months preceding treatment and the six months following treatment. Columns 4 and 7 display the mean number and standard deviation $(S D)$ of ER visits and inpatient nights among those who reported any utilization.

\subsubsection{ER visits}

In the six months prior to entering residential treatment for co-occurring substance use and mental health disorders, $26 \%$ of the 1,394 participants made at least one visit to the ER for medical problems, $13 \%$ for mental health problems, and $23 \%$ for substance use problems. In the six months following treatment, the proportion of patients who made an ER visit was $17 \%$ for medical problems, $5 \%$ for mental health problems, and $7 \%$ for substance use problems. These reductions were statistically significant with $p<.001$. The magnitude of the reductions were $34 \%$ for individuals who reported an ER visit for medical problems $62 \%$, for individuals who reported an ER visit for mental health problems, and 68\% for individuals who reported an ER visit for substance use problems. Further, $45 \%(n=627)$ of patients reported an ER visit for any reason in the pre-treatment period and $24 \%$ $(n=332)$ reported an ER visit for any reason in the post-treatment period, a statistically significant reduction $\left(\chi^{2}=138.33, p<.001\right)$. Though the number and proportion of individuals who reported an ER visit decreased in the post-treatment period, the mean number of visits remained equivalent.

\subsubsection{Inpatient days}

In the six months prior to entering residential treatment for co-occurring substance use and mental health disorders, $11 \%$ of the 1,394 participants reported at least one inpatient day for medical problems, $10 \%$ for mental health problems, and $15 \%$ for substance use problems. In the six months following treatment, the proportion of patients who reported at least one inpatient day was $8 \%$ for medical problems, $3 \%$ for mental health problems, and 5\% for substance use problems. These reductions were statistically significant with $p<.001$. The magnitude of the reductions were $31 \%$ for individuals who reported at least one inpatient day for medical problems $67 \%$ for individuals who reported at least one inpatient day for mental health problems, and $69 \%$ for individuals who reported at least one inpatient day for substance use problems. Further, 26\% $(n=368)$ of patients reported an inpatient stay for any reason in the pre-treatment period and $13 \%(\mathrm{n}=174)$ reported an inpatient stay for any reason in the post-treatment period, a statistically significant reduction $\left(\chi^{2}=86.20\right.$, $p<.001)$. Though the number and proportion of individuals who reported an inpatient stay decreased in the post-treatment period, the mean number of visits remained equivalent.

Table 1. Number of participants with ER visits and inpatient stays for physical, mental health, and substance use problems before and after treatment and mean number of ER visits/inpatient days $(\mathrm{n}=1,394)$

\begin{tabular}{|c|c|c|c|c|c|c|}
\hline & \multicolumn{3}{|c|}{ Pre-Treatment } & \multicolumn{3}{|c|}{ Post-Treatment } \\
\hline & n (\%) & Range & Mean $(S D)^{*}$ & n (\%) & Range & Mean $(S D)^{*}$ \\
\hline \multicolumn{7}{|c|}{ Emergency Room Visits } \\
\hline - Medical $^{\S}$ & $358(26 \%)$ & $1-15$ & $1.88(1.77)$ & 237 (17\%) & $1-30$ & $1.80(2.43)$ \\
\hline - Mental Health ${ }^{\S}$ & $186(13 \%)$ & $1-15$ & $1.71(1.49)$ & $71(5 \%)$ & $1-30$ & $2.30(4.02)$ \\
\hline - Substance Use ${ }^{\S}$ & 319 (23\%) & $1-20$ & 1.79 (1.99) & $101(7 \%)$ & $1-50$ & $2.58(5.57)$ \\
\hline \multicolumn{7}{|l|}{ Hospital Stays } \\
\hline - Medical $^{\S}$ & 159 (11\%) & $1-60$ & $3.48(6.69)$ & 109 (8\%) & $1-32$ & $4.29(5.31)$ \\
\hline - Mental Health ${ }^{\S}$ & $145(10 \%)$ & $1-42$ & $3.37(5.51)$ & 48 (3\%) & $1-27$ & $4.81(5.45)$ \\
\hline - Substance Use $\mathrm{S}^{\S}$ & $208(15 \%)$ & $1-42$ & $3.10(4.47)$ & $64(5 \%)$ & $1-20$ & $3.91(4.58)$ \\
\hline
\end{tabular}

${ }^{\S}$ Chi-square test comparing pre-treatment and post-treatment utilization is significant at $p<.001 ;{ }^{*}$ Mean ER visits/Inpatient stays among patients who reported any visits/stays

\subsection{Healthcare costs}

\subsubsection{ER costs}

Table 2 displays the estimated healthcare costs reported by participants for the six months prior to treatment and the six months following treatment, presented separately for medical, mental health, and substance use. Total pre-treatment ER costs were estimated at $\$ 2,463,240$, while post-treatment
ER costs were estimated to be $\$ 1,342,150$; a $46 \%$ reduction. Comparison of pre-treatment $($ mean $=1,767, S D=3,734)$ and post-treatment (mean $=963, S D=4,468)$ means for total ER costs was statistically significant $(t=5.157, p<.05)$. The average estimated cost per patient for ER visits ranged $\$ 360$ - \$761 in the pre-treatment period and reduced to $\$ 185$ - \$483 in the post-treatment period. A statistically 
significant reduction was demonstrated in each domain.

\subsubsection{Inpatient costs}

The average estimated cost per patient for inpatient stays ranged $\$ 776$ - \$1,023 in the pre-treatment period and reduced to $\$ 367$ - $\$ 743$ in the post-treatment period. The estimated costs from ER visits and hospital stays were significantly re- duced in the six-month post-treatment period across all three domains. Total pre-treatment inpatient costs were estimated to be $\$ 5,888,344$, while post-treatment inpatient costs were estimated at $\$ 2,245,180$; a $62 \%$ reduction. Comparison of pre-treatment (mean $=2,677, S D=9,760)$ and post-treatment (mean $=1,506, S D=6,636)$ means for total inpatient costs was statistically significant $(t=3.705, p<.05)$.

Table 2. Estimated costs of ER visits and inpatient nights, pre-treatment and post-treatment $(\mathrm{n}=1,394)$

\begin{tabular}{|c|c|c|c|c|c|c|}
\hline & \multicolumn{3}{|c|}{ Pre-Treatment } & \multicolumn{3}{|c|}{ Post-Treatment } \\
\hline & Visits/Nights & Total Cost (\$) & Mean $(S D)$ & Visits/Nights & Total Cost (\$) & Mean $(S D)$ \\
\hline \multicolumn{7}{|c|}{ Emergency Room Visits } \\
\hline - Medical $^{*}$ & 672 & $1,061,088$ & $761(1,920)$ & 426 & 672,654 & $483(1,905)$ \\
\hline - Mental Health * & 318 & 502,122 & $360(1,255)$ & 163 & 257,377 & $185(1,630)$ \\
\hline - Substance Use * & 570 & 900,030 & $646(1,913)$ & 261 & 412,119 & $296(2,584)$ \\
\hline \multicolumn{7}{|l|}{ Hospital Stays } \\
\hline - Medical ${ }^{*}$ & 553 & $1,223,236$ & $878(5,551)$ & 468 & $1,035,216$ & $743(4,149)$ \\
\hline - Mental Health * & 489 & $1,081,668$ & $776(4,469)$ & 231 & 510,972 & $367(2,946)$ \\
\hline - Substance Use * & 645 & $1,426,740$ & $1,023(4,525)$ & 251 & 555,212 & $397(2,812)$ \\
\hline
\end{tabular}

${ }^{*} T$-test comparing pre-treatment and post-treatment costs is significant at $p<.001$; Visits/Nights refers to the total number of ER visits or inpatient nights reported by participants; Total cost refers to the total cost of ER visits and inpatient nights for each category; Mean $(S D)$ refers to the mean and standard deviation of the costs per patient averaged across all patients

\section{Discussion}

Literature suggests that individuals with substance use and mental health disorders have higher rates of physical illness and not only utilize more health care than the general populations, but also utilize more costly levels of care such as ER visits at a higher rate. ${ }^{[2,3,5,6,8]}$ Utilizing the ER as entry level into care is not only more costly than a primary care visit, but also associated with delayed medical care which can further drive costs upward. ${ }^{[19,20]}$

There is a great disparity between the amount spent on managing the health care impact of substance abuse and the actual treatment of substance abuse, with managing the impact receiving the lion's share of such spending. ${ }^{[4]}$ Our research focused on determining if enrollment in treatment for substance use and co-occurring mental health disorder impacted the utilization of ER services and hospital overnight stays We looked at three specific types of services within each of these settings: medical, mental health and substance use. Significant savings were noted across all three types of services and both levels of care.

As one might anticipate, the greatest impact was in the use of the ER and hospital overnight stays for substance use problems. Patients who participated in residential substance use disorder treatment were significantly less likely to seek substance use disorder care through the ER or hospital admission following treatment which resulted in a savings over pretreatment costs of nearly $\$ 1.4$ million dollars in the six months following treatment for these services alone. Significant savings were also noted for mental health disorder services of $\$ 815,441$ across the two levels of care. Medical savings were also significant at over half a million dollars. Total savings accrued across both levels of care for all three services were more than $\$ 2.7$ million dollars.

These savings were recognized for the entire sample of respondents, regardless of completion of treatment, relapse, relapse severity or the use of continuing care and aftercare services. These analyses indicate that attending treatment to address substance abuse and mental health issues can play a significant role in reducing the use of expensive acute care such as ER visits and overnight stays in hospitals.

There are limitations to the conclusions that can be drawn from this study. First, the data are collected from patient responses. More reliable data would be available from insurance claim data, and efforts are underway to support a collaborative study. Secondly, it is impossible to know the actual results of those who failed to respond to the survey. Here again, insurance claim data would improve the reliability of the findings. Finally, there is no information about whether other, less costly health care services were utilized and, if so, if they were they utilized more than prior to treatment.

Despite the limitations, these findings represent important new information in a population that has not been thoroughly 
studied. Private pay patients appear to utilize significantly fewer ER and overnight hospital services overall after attend-

\section{REFERENCES}

[1] National Institute on Drug Abuse. Trends \& Statistics. 2016. Available from: https://www.drugabuse.gov/related-topics/ trends-statistics

[2] Mark TL, Woody GE, Juday T, et al. The economic costs of heroin addiction in the United States. Drug and Alcohol Dependence. 2001; 61(2): 195-206. http://dx.doi.org/10.1016/S0376-8 $716(00) 00162-9$

[3] Clark RE, Connell EO, Samnaliev M. Substance Abuse and Healthcare Costs Knowledge Asset, Web site created by the Robert Wood Johnson Foundation's Substance Abuse Policy Research Program. March 2010. Available from: http://saprp.org/knowledgeass ets

[4] CASA Columbia University. Shoveling up II: The Impact of Substance Abuse on Federal, State and Local Budgets. New York; 2009. Available from: http://www.casacolumbia.org

[5] Cederbaum JA, Guerrero EG, Mitchell KR, et al. Utilization of emergency and hospital services among individuals in substance abuse treatment. Substance Abuse Treatment, Prevention and Policy. 2014; 9: 16. http://dx.doi.org/10.1186/1747-597X-9-16

[6] McGeary KA, French MT. Illicit drug use and emergency room utilization. Health Services Research. 2000; 35(1 Pt 1): 153-169. PMid: 10778828.

[7] Substance Abuse and Mental Health Services Administration (SAMHSA), Center for Behavioral Health Statistics and Quality (formerly the Office of Applied Studies). The DAWN Report: Highlights of the 2009 Drug Abuse Warning Network (DAWN) Findings on Drug-Related Emergency Department Visits. 2010. Available from: https://www.drugabuse.gov/publications/drugfac ts/drug-related-hospital-emergency-room-visits

[8] Shanahan CW, Beers D, Alford DP, et al. A Transitional Opioid Program to Engage Hospitalized Drug Users. Journal of General Internal Medicine. 2010; 25(8): 803-808. http://dx.doi.org/10.1007 /s11606-010-1311-3

[9] Substance Abuse and Mental Health Services Administration. Results from the 2013 National Survey on Drug Use and Health: Summary of National Findings, NSDUH Series H-48, HHS Publication No. (SMA) 14-4863. Rockville, MD: Substance Abuse and Mental Health Services Administration; 2014.

[10] Crane EH. The CBHSQ Report: Emergency Department Visits Involving Narcotic Pain Relievers. Rockville, MD: Substance Abuse and Mental Health Services Administration, Center for Behavioral Health Statistics and Quality. 2015. ing residential substance abuse and mental health treatment.

\section{CONFlicts OF INTEREST Disclosure}

The authors declare they have no conflicts of interest.

[11] Owens PL, Barrett ML, Weisrr AJ, et al. Hospital inpatient utilization related to opioid overuse among adults, 1993-2012: HCUP Statistical Brief \#177. Agency for Healthcare Research and Quality, Rockville, MD. 2014.

[12] Bourchery EE, Harwood HJ, Sacks JJ, et al. Economic costs of excessive alcohol consumption in the US, 2006. American Journal of Preventive Medicine. 2011; 41(5): 516-524. PMid: 22011424 http://dx.doi.org/10.1016/j.amepre.2011.06.045

[13] Center for Behavioral Health Statistics and Quality. Behavioral health trends in the United States: Results from the 2014 National Survey on Drug Use and Health (HHS Publication No. SMA 15-4927, NSDUH Series H-50). 2015. Available from: http://www. samhsa.gov/d atal

[14] Guo JJ, Keck PE, Li H, et al. Treatment Costs and Health Care Utilization for Patients with Bipolar Disorder in a Large Managed Care Population. Value in Health. 2008; 11(3): 416-423.

[15] McClellan AT, Kushner H, Metzger D, et al. The fifth edition of the Addiction Severity Index. Journal of Substance Abuse Treatment. 1992; 9: 199-213. http://dx.doi.org/10.1016/0740-5 472 (92) 90062-S

[16] McLellan AT, Alterman AI, Cacciloa J, et al. A new measure of substance abuse treatment: Initial studies of the Treatment Service Review. Journal of Nervous and Mental Disease. 1992; 180: 101-110. PMid: 1737971. http://dx.doi.org/10.1097/00005053-199 202000-00007

[17] Agency for Healthcare Research and Quality. Medical expenditure panel survey: Emergency room services-median and mean expenses per person with expense and distribution of expenses by source of payment. 2016. Available from: https://meps .ahrq.gov/meps web/data_stats/tables_compendia_hh_interactive.jsp

[18] Kaiser Family Foundation. State health facts: Hospital adjusted expenses per inpatient day. 2016. Available from: http://kff.org/ other/state-indicator/expenses-per-inpatient-day/

[19] McWilliams A, Tapp H, Barker J, et al. Cost analysis of the use of emergency departments for primary care services in Charlotte, North Carolina. North Carolina Medical Journal. 2011; 72(4): 265-71. PMid: 22128684.

[20] American College of Emergency Physicians. Insurance Industry Drives Patients to Sacrifice Necessary Medical Care. October 26, 2015. Available from: http://newsroom . acep.org/2015-10-2 6-Insurance-Industry-Drives-Patients-to-Sacrifice -Necessary-Medical-Care 